\title{
Inventário florístico de um remanescente de Floresta Estacional Semidecidual no Sul do Espírito Santo, Brasil: Parque Estadual Cachoeira da Fumaça
}

\author{
Floristic inventory in a Seasonal Semideciduous Forest remnant \\ in Southern Espírito Santo, Brazil: \\ Parque Estadual da Cachoeira da Fumaça
}

\author{
Katiuss Ferreira Borges ${ }^{1}$ \& Michaele Alvim Milward de Azevedo ${ }^{2}$
}

\begin{abstract}
Resumo
Um inventário florístico de espécies de angiospermas provenientes do Parque Estadual Cachoeira da Fumaça (PECF), Espírito Santo, Brasil é apresentado. O PECF está localizado na divisa dos municípios de Alegre e Ibitirama, situado na região do Caparaó, sul do Espírito Santo, protegendo nascentes e remanescentes de um trecho conservado do rio Braço Norte Direito. Objetivou-se com este trabalho ampliar o conhecimento da diversidade de angiospermas de um remanescente de Floresta Atlântica localizado no Sul do Espírito Santo, por meio do levantamento em campo das espécies herbáceas, trepadeiras, arbustivas e arbóreas ocorrentes no PECF. As coletas foram realizadas mensalmente durante janeiro de 2010 a janeiro de 2011 e as amostras botânicas foram depositadas no herbário VIES (subcuradoria Alegre). Os resultados estão apresentados em forma de lista com um total de 222 espécies distribuídas em 171 gêneros e 60 famílias, deste total, 30 espécies são endêmicas do Brasil, quatro estão na lista vermelha de espécies ameaçadas, e 13 são citadas como novas ocorrências para o Espírito Santo. Fabaceae, Asteraceae, Piperaceae, Rubiaceae, Euphorbiaceae, Poaceae, Melastomataceae e Myrtaceae são as famílias mais ricas em número de espécies. O número de táxons encontrados no PECF reafirma que pequenos remanescentes florestais apresentam elevada diversidade de espécies.
\end{abstract}

Palavras-chave: Floresta Atlântica, lista vermelha, novas ocorrências.

\begin{abstract}
This paper provides a floristic inventory of Angiosperms reported from Parque Estadual Cachoeira da Fumaça (PECF), Espírito Santo, Brazil. The PECF is located between Alegre and Ibitirama, region of Caparaó, South of Espírito Santo, protecting springs and remnants of a preserved section of the Braço Norte Direito river, affluent of the Itapemirim river. The objective of this work was to increase the knowledge of the angiosperm diversity of a remnant of the Atlantic Forest located in the south of Espírito Santo, through the field survey of herbaceous, climbing, shrub and tree species occurring in the PECF. The inventory was performed with fortnightly fieldwork from January 2010 to January 2011. The samples collected are available at the herbarium VIES (Alegre). A checklist with 222 species, from 171 genera and 60 families is provided, of this total, 30 species are endemic to Brazil, four are on the red list of endangered species, and 13 are cited as new occurrences for the Espírito Santo. Fabaceae, Asteraceae, Piperaceae, Rubiaceae, Euphorbiaceae, Poaceae, Melastomataceae and Myrtaceae presented the greatest species richness. The number of taxa found in the PECF reaffirms that small remnants of forest present a high diversity of species.
\end{abstract}

Key words: Atlantic Forest, red list, new occurrences.

\footnotetext{
${ }^{1}$ Universidade Federal do Espírito Santo, Centro de Ciências Agrárias e Engenharias, Alto Universitário s/n, Guararema, 29500-000, Alegre, ES, Brasil. katiborgesbio@gmail.com

${ }^{2}$ Universidade Federal Rural do Rio de Janeiro, Inst. Três Rios, Depto. Ciências do Meio Ambiente, Av. Prefeito Alberto Lavinas 1847, Centro, 25802-100, Três Rios, RJ, Brasil. michaelemilward@gmail.com

${ }^{3}$ Autor para correspondência: michaelemilward@gmail.com
} 


\section{Introdução}

Informações sobre a biodiversidade são essenciais para uma ampla gama de usos e tomadas de decisões no âmbito científico, educacional e governamental. Diversos acordos internacionais postulam que sejam realizadas levantamentos e listas de espécies, a fim de conhecer a biodiversidade (Canhos et al. 2004). Para realização de levantamentos com objetivo de classificação de flora, podem ser utilizados dois tipos de sistemas, florísticos ou fisionômicos, os quais fornecem ordenação sistemática dos padrões da vegetação (Lima et al. 2009).

Os sistemas florísticos, partem de espécies frequentes e dominantes, e mostram padrões mais delineados. Para o conhecimento da composição vegetal, são realizados inventários florísticos, os quais permitem a construção de bancos de informações, por meio, principalmente, de exsicatas depositadas em herbários e assim fornecem informações de flora de uma determinada área. Esses dados podem vir a ser utilizados em diferentes estudos, como ecológicos, taxonômicos, geográficos e de recuperação de ambientes degradados (Souza et al. 2009).

As florestas tropicais da América do Sul apresentam abundante diversidade de espécies. Algumas são referenciadas como as mais diversas do mundo (Martini et al. 2007; Saiter et al. 2008). Essa elevada diversidade de flora vem sendo associada com a heterogeneidade em diferentes escalas espaciais (Pitman et al. 2002; Hernández et al. 2012).

Entre elas destaca-se a Floresta Atlântica, a qual é considerada uma das zonas biogeográficas mais abundantes em número de espécies vegetais do mundo, sendo a segunda maior floresta tropical existente no continente americano e um dos 25 hotspots de biodversidade mundial (Myers et al. 2000; Tabarelli et al. 2005). No Brasil, ela chegou a cobrir uma área correspondente a 1-1,5 milhões de $\mathrm{km}^{2}$ (Galindo-Leal \& Câmara 2005). A prática de desmatamento durante os séculos promoveu uma redução deste bioma para $12-17 \%$ da sua cobertura original, levando à formação de fragmentos florestais, onde mais de $80 \%$ apresentam cerca de 50 ha (Ribeiro et al. 2009). O tamanho e forma dos remanescentes afeta de forma significativa a diversidade de espécies, devido ao grau de conectividade entre eles (Metzger 2003).

O estado do Espírito Santo, apesar de possuir área relativamente pequena, quando comparado a outros estados, é o décimo em riqueza de espécies vegetais (BFG 2015). Nesse montante, muitas espécies são restritas e atualmente classificadas como ameaçadas de extinção (Martinelli \& Moraes 2013; BFG 2015). A ampla gama de espécies encontradas, é evidenciada pelas diferentes tipologias topográficas e climáticas, ocupando desde porções ao nível do mar até elevadas altitudes, e também diversos tipos vegetacionais encontrados: restingas, manguezais, florestas semideciduais, floresta ombrófilas e refúgios de vegetação (Pereira 2007; Metzger 2009).

Embora, nos últimos anos tenham sido desenvolvidos estudos florísticos para o estado, como por exemplo: Pereira et al. (1998); Pereira \& Araújo (2000); Assis et al. (2004); Fraga et al. (2007); Fabris \& Peixoto (2013); Oliveira et al. (2013); Chagas et al. (2014); Saiter \& Thomaz (2014); Sarnaglia Junior et al. (2014); Dutra et al. (2015), Peterle et al. (2015); Luber et al. (2016), Souza et al. (2016) e Couto et al. (2017), a listagem da biodiversidade vegetal para o estado continua defasada, pois não são todos os remanescentes florestais que foram objetos de estudos e contemplados com o inventário florístico.

Um exemplo é o Parque Estadual da Cachoeira da Fumaça (PECF), área de conservação que possui nascentes e remanescentes de um trecho conservado do rio Braço Norte Direito, afluente do rio Itapemirim. A região é formada por fragmentos florestais de mata ciliar, vegetação rupestre, brejo herbáceo, vegetação exótica, estágio inicial, médio e avançado de regeneração da Floresta Atlântica e agricultura. O PECF foi criado em 1990, contemplando uma área de 24,2 ha. Porém no ano de 2009, a área do parque foi ampliada para 162,5 ha (IDAF 2000; IEMA 2017). De acordo com o Sistema Nacional de Unidades de Conservação da Natureza - SNUC, parques nacionais, estaduais e municipais tem como objetivo básico preservar os ecossistemas naturais de grande relevância ecológica, permitindo a realização de pesquisas científicas e o desenvolvimento de atividades de educação e interpretação ambiental, de recreação em contato com a natureza e de turismo ecológico.

A riqueza da biodiversidade e ausência de coletas botânicas nos fragmentos de Mata Atlântica da região Sul do Espírito Santo, em especial o PECF, indicam a necessidade de se conhecer a flora local, podendo auxiliar a recuperação de ambientes degradados nos arredores do PECF, além de fornecer subsídio para projetos de conservação, proteção e preservação ambiental.

Diante do exposto, este trabalho teve por objetivo ampliar o conhecimento da diversidade de angiospermas do PECF, uma importante unidade de conservação pertencente a um remanescente 
de Floresta Atlântica localizado no Sul do Espírito Santo, por meio do levantamento em campo das espécies herbáceas, trepadeiras, arbustivas e arbóreas.

\section{Material e Métodos}

O PECF (Fig. 1) localiza-se na divisa entre os municípios de Ibitirama e Alegre, alocados na Região do Caparaó, no estado do Espírito Santo, entre as coordenadas $40^{\circ} 50^{\prime}-41^{\circ} 50^{\prime} \mathrm{O}$ e $20^{\circ} 10^{\prime}$ $21^{\circ} 5^{\prime} \mathrm{S}$ e apresenta uma área de 162,5 ha. Encontrase sob domínio de Floresta Atlântica, com formação florestal do tipo Floresta Estacional Semidecidual. O clima é tropical, com pluviosidade média anual de $1.200 \mathrm{~mm}$, sendo o período chuvoso de novembro a março e o de seca de maio a setembro e a temperatura média fica em torno de $23^{\circ} \mathrm{C}$ (IDAF, 2000; IEMA 2017).

Para realização do inventário florístico foram realizadas coletas de campo mensais, onde foram realizados caminhamentos nas trilhas e no interior dos fragmentos de mata, no período de janeiro de
2010 a janeiro de 2011 em diferentes pontos do PECF a fim de coletar o material vegetal em estágio reprodutivo. O estágio de regeneração dos fragmentos vegetacionais foi definido segundo a Resolução $n^{\circ}$ 29 (CONAMA 1994). As amostras coletadas foram herborizadas seguindo técnicas usuais em taxonomia vegetal e incluídas no herbário VIES, subcuradoria Alegre, com duplicatas enviadas aos herbários R, RB, SPF, UB e UEC, os acrônimos estão de acordo com Thiers (continuamente atualizado). Para identificação dos materiais coletados foram realizadas comparações com materiais disponíveis em herbários, consultas à material bibliográfico disponível e envio de exsicatas para especialistas. Os nomes atualmente aceitos foram verificados no sítio eletrônico do The Plant List ( $<$ http://www. theplantlist.org/>).

A confecção da lista de florística contou apenas com os espécimes identificados minimamente em nível de gênero. Seguiu-se o que foi proposto por Font Quer (1992), para definição da forma de vida.

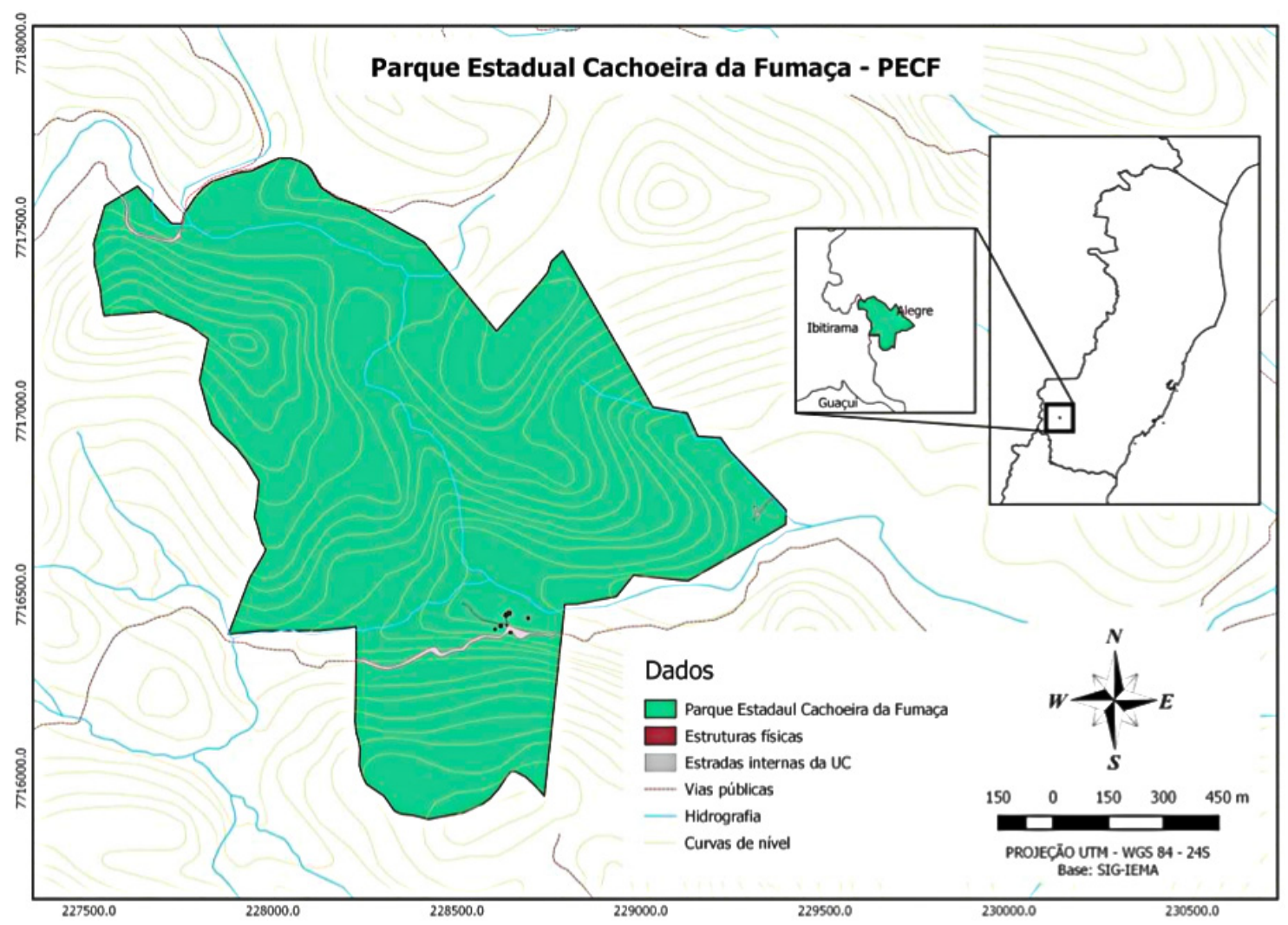

Figura 1 - Mapa da área total do Parque Estadual da Cachoeira da Fumaça, Alegre e Ibitirama, Espírito Santo, Brasil, delimitado em verde, retirado de IEMA 2017.

Figure 1 - Map of the total area of Parque Estadual da Cachoeira da Fumaça, Alegre and Ibitirama, Espírito Santo, Brazil, delimited in green, taken from IEMA 2017. 
A distribuição geográfica, o tipo de fitofisionomia presente, e se as espécies eram endêmicas ou não, foi pesquisado no BFG (2015).

Foi considerada nova ocorrência de uma espécie para o estado do Espírito Santo, quando a mesma não estava listada em: Pereira et al. 1998; Pereira \& Araújo 2000; Assis et al. 2004; Fraga et al. 2007; Fabris \& Peixoto 2013; Oliveira et al. 2013; Chagas et al. 2014; Saiter \& Thomaz 2014; Sarnaglia Junior et al. 2014; Dutra et al. 2015; Peterle et al. 2015; Luber et al. 2016; Souza et al. 2016 e Couto et al. 2017, e no sítio eletrônico do Species Link (<http://splink.cria.org.br/>) e/ou no BFG (2015) ocorrentes no Espírito Santo.

O grau de ameaça das espécies de acordo com os critérios da União Internacional para a Conservação da Natureza (IUCN) foram avaliados pela lista de espécies ameaçadas disponível pelo Ministério do Meio Ambiente (MMA 2014) e no sítio eletrônico do Centro Nacional de Conservação da Flora (CNCFlora 2017).

\section{Resultados e Discussões}

Após minuciosa análise das amostras vegetais coletadas, foram verificadas 222 espécies, pertencentes a 171 gêneros e 60 famílias (Tab. 1). Deste total, $80,18 \%$ correspondem a espécies nativas da Mata Atlântica, 5,4\% espécies exóticas da Mata Atlântica, 5,86\% espécies exóticas brasileiras e $8,56 \%$ espécies naturalizadas.

Foram registradas 27 novas ocorrências para o Espírito Santo, destas, 13 são nativas da Mata Atlântica: Caesalpinia peltophoroides Benth., Coccocypselum aureum (Spreng.) Cham. \& Schltdl., Cuphea gracilis Kunth, Cyperus difformis L., Dasyanthina serrata (Less.) H. Rob., Heliconia densiflora B. Verl., Manettia pubescens Cham. \& Schltdl., Myrcia floridissima Sobral, Oxypetalum harleyi (Fontella \& Goyder) Farinaccio, Piper frutescens C. DC., Senegalia parviceps (Speg.) Seigler \& Ebinger, Syagrus oleracea (Mart.) Becc. e Vernonanthura polyantes (Sprengel) Vega \& Dematteis. As demais novas ocorrências são representadas por seis espécies exóticas da Mata Atlântica, cinco exóticas aos ecossistemas brasileiros e três naturalizadas (Tab. 1). Estas 14 espécies introduzidas devem-se provavelmente pela área do PECF estar rodeadas por fazendas ou sítios.

Entre todas as espécies, 30 são endêmicas do Brasil, três apresentam grau de ameaça vulnerável de acordo com a IUCN: Cedrela fissilis Vell., Euterpe edulis Mart. e Rudgea jasminioides (Cham.) Müll. Arg., e uma menos preocupante: Xylopia aromatica
(Lam.) Mart., sendo encontradas na lista vermelha de espécies ameaçadas (Tab. 1).

Quanto às espécies endêmicas, o estado do Espírito Santo possui 564 espécies, equivalendo a 10,6\% (BFG 2015), enquanto que o PECF mostrou uma porcentagem relativa maior do que a ocorrida para o estado, onde das 222 angiospermas encontradas 30 são endêmicas, ou seja, 13,5\% das espécies são endêmicas do Brasil. Em todo o mundo, o número de espécies endêmicas tem crescido. De 2010 para 2015 houve um acréscimo de $1 \%$, com adição de 791 novas espécies endêmicas (BFG 2015). Remanescentes onde encontram-se espécies endêmicas são importantes na biogeografia e devem ser priorizadas na seleção de áreas para conservação (Brooks et al. 1992).

As famílias com maior número de espécies foram Fabaceae (29 ssp.); Asteraceae (24 ssp.); Piperaceae e Rubiaceae (dez spp.); Euphorbiaceae e Poaceae (nove spp.); Melastomataceae e Myrtaceae (oito ssp.), correspondendo a cerca de $48 \%$ das espécies levantadas (Tab. 1). Com exceção da família Piperaceae, as demais, estão entre as dez famílias mais representativas da Flora do Brasil (BFG 2015).

A riqueza encontrada para Fabaceae e Asteraceae pode ser explicada pelo caráter pioneiro que suas espécies apresentam (Pillar et al. 2009), pois são consideradas como indicadores de processo de regeneração (Saiter et al. 2007), como visualizado em diversos pontos do PECF.

Entre as espécies puderam ser encontradas algumas com valor ornamental, como por exemplo Hedychium coronarium J. Köing, Heliconia spp., Pticairnia flammea Lindl. e Thumbergia alata Bojer ex. Sims.; medicinal: Baccharis crispa Spreng., Eupatorium maximiliani Schrader ex DC., Passiflora alata Curtis, Passiflora edulis Sims, Plantago major L., Polygala cyparissias A. St.-Hil. \& Moq.; e com interesse econômico: Anadenanthera macrocarpa (Benth.) Brenan, Cedrela fissilis Vell., Coffea sp., Euterpe edulis, Passiflora alata, Passiflora edulis e Phaseolus vulgaris L. A presença de algumas dessas espécies, podem ser explicadas pela presença de propriedades agrícolas no entorno do PECF.

As espécies exóticas brasileiras e as naturalizadas, representam $15 \%$ do total das espécies registradas, sendo consideradas espécies invasoras, responsáveis por causar impactos bióticos e abióticos que interferem na conservação da diversidade e dos ecossistemas (Sampaio \& Schmidt 2013).

No estudo realizado por Sampaio \& Schmidt (2013), sobre as espécies exóticas invasoras (EEI) encontradas em Unidades de Conservação, os autores 
Tabela 1 - Composição florística com respectivo hábito das espécies do Parque Estadual da Cachoeira da Fumaça, Alegre/Ibitirama, Espírito Santo, Brasil. Legenda: * = espécies catalogadas pela primeira vez para o estado do Espírito Santo; $\#$ = espécies endêmicas; $\Delta$ = espécies com algum grau de ameaça de acordo com os critérios da IUCN. Hábito: $\mathrm{AR}=$ arbóreo; $\mathrm{HE}=$ herbáceo; $\mathrm{AB}=$ arbustivo; $\mathrm{SB}=$ subarbustivo; $\mathrm{TP}=$ trepadeira; $\mathrm{LI}=$ liana. Origem: EX-BR = Exótica Ecossistemas Brasileiros; EX-MA = Exótica Mata Atlântica; NMA = Nativa Mata Atlântica; NAT = Naturalizada. Material depositado no herbário VIES, subcuradoria Alegre.

Table 1 - Floristic composition with respective habit of the species from Parque Estadual da Cachoeira da Fumaça, Alegre/Ibitirama, Espírito Santo, Brazil. Legend: $*=$ species cataloged for the first time for the state of Espírito Santo; \#= endemic species; $\Delta=$ species with some degree of threat according to IUCN criteria. Habit: $\mathrm{AR}=$ arboreal; HE = herbaceous; $\mathrm{AB}=$ shrub; $\mathrm{SB}=$ sub-shrub; TP = climber; LI = liana. Origin: EX-BR = Exotic Brazilian Ecosystems; EX-MA = Exotic Atlantic Forest; NMA = Native Atlantic Forest; NAT $=$ Naturalized. Material deposited in the herbarium VIES (Alegre).

\begin{tabular}{|c|c|c|c|c|}
\hline Família & Gênero / Espécie & Hábito & Origem & Voucher \\
\hline \multirow[t]{3}{*}{ Acanthaceae } & Ruellia L. sp. & $\mathrm{HE}$ & NMA & M.A. Milward-de-Azevedo et al. 344 \\
\hline & Thunbergia alata Bojer ex. Sims. & $\mathrm{HE}$ & NAT & M.A. Milward-de-Azevedo et al. 254 \\
\hline & Whitfieldia Hooker sp. & $\mathrm{HE}$ & EX-BR & M.A. Milward-de-Azevedo et al. s.n. \\
\hline Achariaceae & Carpotroche brasiliensis (Raddi) A. Gray & AR & NMA & M.A. Milward-de-Azevedo et al. 309 \\
\hline \multirow[t]{3}{*}{ Anacardiaceae } & Anacardium occidentale L. & AR & NMA & M.A. Milward-de-Azevedo et al. s.n. \\
\hline & Mangifera indica $\mathrm{L}$. & AR & EX-BR & M.A. Milward-de-Azevedo et al. s.n. \\
\hline & Spondias mombin L. & $\mathrm{AR}$ & NMA & M.A. Milward-de-Azevedo et al. s.n. \\
\hline \multirow[t]{4}{*}{ Annonaceae } & Annona muricata $\mathrm{L}$. & AR & EX-BR & M.A. Milward-de-Azevedo et al. s.n. \\
\hline & Guatteria ferruginea A.St.-Hil. & $\mathrm{AR}$ & NMA & M.A. Milward-de-Azevedo et al. 466 \\
\hline & \# Guatteria pogonopus Mart. & AR & NMA & M.A. Milward-de-Azevedo et al. s.n. \\
\hline & $\Delta$ Xylopia aromatica (Lam.) Mart. & $\mathrm{AB}$ & NMA & M.A. Milward-de-Azevedo et al. 407 \\
\hline \multirow[t]{5}{*}{ Apocynaceae } & \# Allamanda polyantha Müll. Arg. & SB & NMA & M.A. Milward-de-Azevedo et al. s.n. \\
\hline & Asclepias curassavica L. & $\mathrm{HE}$ & NMA & M.A. Milward-de-Azevedo et al. s.n. \\
\hline & $\begin{array}{l}\text { \#* Oxypetalum harleyi (Fontella \& Goyder) } \\
\text { Farinaccio }\end{array}$ & $\mathrm{TP}$ & NMA & M.A. Milward-de-Azevedo et al. 246 \\
\hline & \# Tabernaemontana hystrix Steud. & AR & NMA & M.A. Milward-de-Azevedo et al. s.n. \\
\hline & \# Tabernaemontana laeta Mart. & $\mathrm{AB}$ & NMA & M.A. Milward-de-Azevedo et al. s.n. \\
\hline Araliaceae & $\begin{array}{l}\text { Dendropanax cuneatus (DC.) } \\
\text { Decne. \& Planch. }\end{array}$ & AR & NMA & M.A. Milward-de-Azevedo et al. 277 \\
\hline \multirow[t]{2}{*}{ Arecaceae } & $\Delta$ Euterpe edulis Mart. & AR & NMA & M.A. Milward-de-Azevedo et al. s.n. \\
\hline & \#* Syagrus oleracea (Mart.) Becc. & AR & NMA & M.A. Milward-de-Azevedo et al. s.n. \\
\hline \multirow[t]{8}{*}{ Asteraceae } & Baccharis crispa Spreng. & $\mathrm{HE}$ & NMA & M.A. Milward-de-Azevedo et al. 289 \\
\hline & Baccharis dracunculifolia DC. & $\mathrm{AB}$ & NMA & M.A. Milward-de-Azevedo et al. s.n. \\
\hline & \# Baccharis serrulata (Lam.) Pers. & $\mathrm{HE}$ & NMA & M.A. Milward-de-Azevedo et al. s.n. \\
\hline & Centratherum punctatum Cass. & $\mathrm{HE}$ & NMA & M.A. Milward-de-Azevedo et al. 224 \\
\hline & Chaptalia Vent. sp. & $\mathrm{HE}$ & NMA & M.A. Milward-de-Azevedo et al. s.n. \\
\hline & $\begin{array}{l}\text { Chromolaena laevigata (Lam.) } \\
\text { R.M. King \& H. Rob. }\end{array}$ & $\mathrm{HE}$ & NMA & $\begin{array}{l}\text { M.A. Milward-de-Azevedo et al. } \\
\text { 217, 220, } 227\end{array}$ \\
\hline & \# Cyrtocymura scorpioides (Lam.) H. Rob. & $\mathrm{HE}$ & NMA & $\begin{array}{l}\text { M.A. Milward-de-Azevedo et al. } \\
216,221,252,290,297,307,325\end{array}$ \\
\hline & * Dasyanthina serrata (Less.) H. Rob. & $\mathrm{HE}$ & NMA & M.A. Milward-de-Azevedo et al. s.n. \\
\hline
\end{tabular}




\begin{tabular}{|c|c|c|c|c|}
\hline Família & Gênero / Espécie & Hábito & Origem & Voucher \\
\hline & Elephantopus L. & $\mathrm{HE}$ & NMA & M.A. Milward-de-Azevedo et al. 241 \\
\hline & Emilia fosbergii Nicolson & $\mathrm{HE}$ & NMA & $\begin{array}{l}\text { M.A. Milward-de-Azevedo et al. } \\
\text { 258, } 288\end{array}$ \\
\hline & Eupatorium maximiliani Schrades ex DC. & $\mathrm{AB}$ & NMA & M.A. Milward-de-Azevedo et al. s.n. \\
\hline & Eupatorium L. sp. 1 & SB & EX-MA & M.A. Milward-de-Azevedo et al. 293 \\
\hline & Eupatorium L. sp. 2 & $\mathrm{HE}$ & EX-MA & M.A. Milward-de-Azevedo et al. s.n. \\
\hline & Heterocondylus R.M. King sp. & $\mathrm{HE}$ & NMA & M.A. Milward-de-Azevedo et al. 321 \\
\hline & Lepidaploa Cass. sp. & $\mathrm{HE}$ & NMA & M.A. Milward-de-Azevedo et al. 290 \\
\hline & Mikania hirsutissima DC. & $\mathrm{SB}$ & NMA & M.A. Milward-de-Azevedo et al. 272 \\
\hline & Spilanthes Jacq. sp. & $\mathrm{HE}$ & EX-MA & M.A. Milward-de-Azevedo et al. s.n. \\
\hline & Stevia oligocephala DC. & $\mathrm{HE}$ & NMA & M.A. Milward-de-Azevedo et al. 278 \\
\hline & $\begin{array}{l}\text { Trixis antimenorrhoea (Schrank) } \\
\text { Mart. ex Kuntze }\end{array}$ & $\mathrm{HE}$ & NMA & M.A. Milward-de-Azevedo et al. 327 \\
\hline & Taraxacum officinale Weber ex F.H. Wigg. & $\mathrm{HE}$ & EX-BR & M.A. Milward-de-Azevedo et al. 228 \\
\hline & Vernonia Schreb. sp. & $\mathrm{AB}$ & NMA & $\begin{array}{l}\text { M.A. Milward-de-Azevedo et al. } \\
\text { 230, } 301\end{array}$ \\
\hline & Vernonia scorpioides (Lam.) Pers. & $\mathrm{HE}$ & EX-MA & $\begin{array}{l}\text { M.A. Milward-de-Azevedo et al. } \\
\text { 216, 221, 252, } 297\end{array}$ \\
\hline & $\begin{array}{l}\text { * Vernonanthura polyantes (Sprengel) } \\
\text { Vega \& Dematteis }\end{array}$ & $\mathrm{AB}$ & NMA & M.A. Milward-de-Azevedo et al. 337 \\
\hline & Wedelia paludosa DC. & $\mathrm{HE}$ & NMA & M.A. Milward-de-Azevedo et al. s.n. \\
\hline \multirow[t]{3}{*}{ Bignoniaceae } & Tecoma stans (L.) Seem. & $\mathrm{AB}$ & NAT & M.A. Milward-de-Azevedo et al. s.n. \\
\hline & Tabebuia heptaphylla (Vell.) Toledo & AR & NMA & M.A. Milward-de-Azevedo et al. s.n. \\
\hline & Tabebuia serratifolia (Vahl) G. Nicholson & $\mathrm{AR}$ & NMA & M.A. Milward-de-Azevedo et al. s.n. \\
\hline Bixaceae & Bixa orellana L. & $\mathrm{AB}$ & NMA & M.A. Milward-de-Azevedo et al. s.n. \\
\hline \multirow[t]{3}{*}{ Boraginaceae } & Cordia curassavica (Jacq.) Roem \& Schult. & $\mathrm{HE}$ & NMA & M.A. Milward-de-Azevedo et al. s.n. \\
\hline & Cordia polycephala (Lam.) I.M. Johnst. & SB & NMA & M.A. Milward-de-Azevedo et al. s.n. \\
\hline & Cordia L. sp. & SB & NMA & M.A. Milward-de-Azevedo et al. 419 \\
\hline \multirow[t]{6}{*}{ Bromeliaceae } & Aechmea Ruiz \& Pavi sp. & $\mathrm{HE}$ & NMA & M.A. Milward-de-Azevedo et al. s.n. \\
\hline & \# Alcantarea Harms sp. & $\mathrm{HE}$ & NMA & M.A. Milward-de-Azevedo et al. s.n. \\
\hline & Billbergia Thunb sp. & $\mathrm{HE}$ & NMA & M.A. Milward-de-Azevedo et al. s.n. \\
\hline & Neoregelia L.B. Sm. sp. & $\mathrm{HE}$ & NMA & M.A. Milward-de-Azevedo et al. s.n. \\
\hline & \# Pitcairnia flammea Lindl. & $\mathrm{HE}$ & NMA & M.A. Milward-de-Azevedo et al. 263 \\
\hline & Vriesea Lindl. sp. & $\mathrm{HE}$ & NMA & M.A. Milward-de-Azevedo et al. s.n. \\
\hline Cactaceae & Epiphyllum phyllanthus (L.) Haw. & $\mathrm{HE}$ & NMA & M.A. Milward-de-Azevedo et al. s.n. \\
\hline Campanulaceae & \# Lobelia fistulosa Vell. & $\mathrm{HE}$ & NMA & M.A. Milward-de-Azevedo et al. 359 \\
\hline Cannabaceae & Trema micrantha (L.) Blume & $\mathrm{AR}$ & NMA & M.A. Milward-de-Azevedo et al. 386 \\
\hline Clethraceae & Clethra scabra Pers. & AR & NMA & M.A. Milward-de-Azevedo et al. s.n. \\
\hline Combretaceae & Terminalia catappa $\mathrm{L}$. & AR & NAT & M.A. Milward-de-Azevedo et al. s.n. \\
\hline
\end{tabular}




\begin{tabular}{|c|c|c|c|c|}
\hline Família & Gênero / Espécie & Hábito & Origem & Voucher \\
\hline & \# Terminalia mameluco Alwan \& Stace & $\mathrm{AR}$ & NMA & M.A. Milward-de-Azevedo et al. s.n. \\
\hline Commelinaceae & Commelina diffusa Burm. F. & $\mathrm{HE}$ & NMA & M.A. Milward-de-Azevedo et al. 463 \\
\hline Convolvulaceae & Ipomoea L. sp. & $\mathrm{TP}$ & NMA & M.A. Milward-de-Azevedo et al. 306 \\
\hline Costaceae & Costus spiralis (Jacq.) Roscoe & $\mathrm{HE}$ & NMA & $\begin{array}{l}\text { M.A. Milward-de-Azevedo et al. } \\
314,435\end{array}$ \\
\hline \multirow[t]{4}{*}{ Cyperaceae } & Cyperus laxus Lam. & $\mathrm{HE}$ & NMA & M.A. Milward-de-Azevedo et al. 367 \\
\hline & * Cyperus difformis $\mathrm{L}$. & $\mathrm{HE}$ & NMA & M.A. Milward-de-Azevedo et al. s.n. \\
\hline & \# Cyperus odoratus $\mathrm{L}$. & $\mathrm{HE}$ & NMA & M.A. Milward-de-Azevedo et al. 292 \\
\hline & Eleocharis R.Br.sp. & $\mathrm{HE}$ & NMA & M.A. Milward-de-Azevedo et al. s.n. \\
\hline Dilleniaceae & Davilla rugosa Poir. & LI & NMA & $\begin{array}{l}\text { M.A. Milward-de-Azevedo et al. } \\
\text { 270, 291, } 353\end{array}$ \\
\hline \multirow[t]{2}{*}{ Erythroxylaceae } & Erythroxylum citrifolium A.St.-Hil. & $\mathrm{AB}$ & NMA & M.A. Milward-de-Azevedo et al. 461 \\
\hline & Erythroxylum deciduum A.St.-Hil. & $\mathrm{AB}$ & NMA & M.A. Milward-de-Azevedo et al. 452 \\
\hline \multirow[t]{9}{*}{ Euphorbiaceae } & Acalypha brasiliensis Müll.Arg. & $\mathrm{AB}$ & NMA & M.A. Milward-de-Azevedo et al. 429 \\
\hline & Aparisthmium Endl. & $\mathrm{AR}$ & NMA & M.A. Milward-de-Azevedo et al. 280 \\
\hline & Aparisthmium cordatum (A.Juss) Baill & $\mathrm{AR}$ & NMA & M.A. Milward-de-Azevedo et al. 459 \\
\hline & Alchornea Sw. Sp. & $\mathrm{AB}$ & NMA & M.A. Milward-de-Azevedo et al. 280 \\
\hline & Croton floribundus Spreng. & $\mathrm{AR}$ & NMA & M.A. Milward-de-Azevedo et al. 283 \\
\hline & Hura crepitans L. & $\mathrm{AR}$ & EX-MA & M.A. Milward-de-Azevedo et al. s.n. \\
\hline & Mabea fistulifera Mart. & $\mathrm{AR}$ & NMA & M.A. Milward-de-Azevedo et al. 304 \\
\hline & \# Manihot pilosa Pohl. & $\mathrm{AB}$ & NMA & M.A. Milward-de-Azevedo et al. 392 \\
\hline & Ricinus communis $\mathrm{L}$. & $\mathrm{AB}$ & EX-BR & M.A. Milward-de-Azevedo et al. s.n. \\
\hline \multirow[t]{16}{*}{ Fabaceae } & \# Acacia plumosa Mart. ex Colla & $\mathrm{AR}$ & NMA & M.A. Milward-de-Azevedo et al. s.n. \\
\hline & \# Albizia polycephala (Benth.) Killip & $\mathrm{AR}$ & NMA & M.A. Milward-de-Azevedo et al. s.n. \\
\hline & Anadenanthera colubrina (Vell.) Brenan & AR & NMA & M.A. Milward-de-Azevedo et al. 410 \\
\hline & Bauhinia monandra Kurz & $\mathrm{AR}$ & NMA & M.A. Milward-de-Azevedo et al. 409 \\
\hline & Caesalpina ferrea Mart. & $\mathrm{AR}$ & NMA & M.A. Milward-de-Azevedo et al. s.n. \\
\hline & \#* Caesalpinia peltophoroides Benth. & $\mathrm{AR}$ & NMA & M.A. Milward-de-Azevedo et al. s.n. \\
\hline & Centrosema brasilianum (L.) Benth. & $\mathrm{HE}$ & NMA & M.A. Milward-de-Azevedo et al. 408 \\
\hline & Clitoria fairchildiana R.A. Howard & $\mathrm{AR}$ & NMA & M.A. Milward-de-Azevedo et al. s.n. \\
\hline & * Crotalaria nitens Kunth & $\mathrm{HE}$ & EX-MA & M.A. Milward-de-Azevedo et al. 238 \\
\hline & Dalbergia L. sp. & $\mathrm{AR}$ & NMA & M.A. Milward-de-Azevedo et al. s.n. \\
\hline & Desmodium uncinatum (Jacq.) DC. & $\mathrm{HE}$ & NMA & $\begin{array}{l}\text { M.A. Milward-de-Azevedo et al. } \\
391,404,440,458\end{array}$ \\
\hline & * Hymenolobium petraeum Ducke & $\mathrm{AR}$ & EX-MA & M.A. Milward-de-Azevedo et al. s.n. \\
\hline & Indigofera suffruticosa Mill. & $\mathrm{AB}$ & NMA & M.A. Milward-de-Azevedo et al. 399 \\
\hline & Inga edulis Mart. & $\mathrm{AR}$ & NMA & M.A. Milward-de-Azevedo et al. s.n. \\
\hline & \# Inga sessilis (Vell.) Mart. & $\mathrm{AR}$ & NMA & M.A. Milward-de-Azevedo et al. s.n. \\
\hline & Inga vera subsp. affinis (DC.) T.D.Penn. & $\mathrm{AR}$ & NMA & M.A. Milward-de-Azevedo et al. 418 \\
\hline
\end{tabular}




\begin{tabular}{|c|c|c|c|c|}
\hline Família & Gênero / Espécie & Hábito & Origem & Voucher \\
\hline & Machaerium hirtum (Vell.) Stellfeld & AR & NMA & M.A. Milward-de-Azevedo et al. s.n. \\
\hline & Machaerium nyctitans (Vell.) Benth. & AR & NMA & M.A. Milward-de-Azevedo et al. s.n. \\
\hline & Mimosa pudica L. & $\mathrm{HE}$ & NMA & M.A. Milward-de-Azevedo et al. s.n. \\
\hline & Parkia pendula (Willd.) Benth. ex Walp. & AR & NMA & M.A. Milward-de-Azevedo et al. s.n. \\
\hline & Peltophorum dubium (Spreng.) Taub. & AR & NMA & M.A. Milward-de-Azevedo et al. s.n. \\
\hline & Phaseolus vulgaris L. & $\mathrm{HE}$ & EX-BR & M.A. Milward-de-Azevedo et al. s.n. \\
\hline & Piptadenia gonoacantha (Mart.) J.F. Macbr. & AR & NMA & M.A. Milward-de-Azevedo et al. s.n. \\
\hline & $\begin{array}{l}\text { Samanea inopinata (Harms) } \\
\text { Barneby \& J.W. Grimes }\end{array}$ & AR & EX-MA & M.A. Milward-de-Azevedo et al. s.n. \\
\hline & Schizolobium parahyba (Vell.) S.F. Blake & AR & NMA & M.A. Milward-de-Azevedo et al. s.n. \\
\hline & $\begin{array}{l}\text { * Senegalia parviceps (Speg.) } \\
\text { Seigler \& Ebinger }\end{array}$ & AR & NMA & M.A. Milward-de-Azevedo et al. s.n. \\
\hline & Senna siamea (Lam.) H.S. Irwin \& Barneby & AR & NAT & M.A. Milward-de-Azevedo et al. 236 \\
\hline & Senna obtusifolia (L.) H.S.Irwin \& Barneby & SB & NMA & M.A. Milward-de-Azevedo et al. 267 \\
\hline & Vigna Savi sp. & $\mathrm{HE}$ & NMA & M.A. Milward-de-Azevedo et al. 250 \\
\hline \multirow[t]{5}{*}{ Heliconiaceae } & * Heliconia acuminata Rich. & $\mathrm{HE}$ & EX-MA & $\begin{array}{l}\text { M.A. Milward-de-Azevedo et al. } \\
312,373\end{array}$ \\
\hline & \# Heliconia farinosa Raddi & $\mathrm{HE}$ & NMA & M.A. Milward-de-Azevedo et al. 274 \\
\hline & Heliconia psittacorum L. & $\mathrm{HE}$ & NMA & M.A. Milward-de-Azevedo et al. s.n. \\
\hline & * Heliconia densiflora B. Verl. & SB & NMA & M.A. Milward-de-Azevedo et al. 259 \\
\hline & Heliconia velloziana L. Emygd. & $\mathrm{HE}$ & NMA & M.A. Milward-de-Azevedo et al. 274 \\
\hline Hypoxidaceae & Hypoxis decumbens L. & $\mathrm{HE}$ & NMA & $\begin{array}{l}\text { M.A. Milward-de-Azevedo et al. } \\
\text { 257, } 279\end{array}$ \\
\hline \multirow[t]{3}{*}{ Lamiaceae } & Mesosphaerum suaveolens (L.) Poit & $\mathrm{SB}$ & NMA & M.A. Milward-de-Azevedo et al. 264 \\
\hline & Ocimum gratissimum $\mathrm{L}$. & SB & NAT & M.A. Milward-de-Azevedo et al. 415 \\
\hline & * Physostegia virginiana (L.) Benth. & $\mathrm{HE}$ & EX-BR & M.A. Milward-de-Azevedo et al. 413 \\
\hline \multirow[t]{2}{*}{ Lecythidaceae } & Bertholletia excelsa Bonpl. & AR & EX-MA & M.A. Milward-de-Azevedo et al. s.n. \\
\hline & Cariniana Casar. sp. & AR & NMA & M.A. Milward-de-Azevedo et al. s.n. \\
\hline Lythraceae & * Cuphea gracilis Kunth & $\mathrm{HE}$ & NMA & $\begin{array}{l}\text { M.A. Milward-de-Azevedo et al. } \\
\text { 260, 281, } 427\end{array}$ \\
\hline \multirow[t]{2}{*}{ Loranthaceae } & Phoradendron Nutt. sp. & $\mathrm{HE}$ & NMA & M.A. Milward-de-Azevedo et al. s.n. \\
\hline & Psittacanthus Mart. sp. & $\mathrm{HE}$ & NMA & M.A. Milward-de-Azevedo et al. s.n. \\
\hline \multirow[t]{2}{*}{ Malpighiaceae } & Peixotoa reticulata Griseb. & $\mathrm{AB}$ & NMA & M.A. Milward-de-Azevedo et al. 232 \\
\hline & Heteropterys nitida (Lam.) DC. & LI & NMA & M.A. Milward-de-Azevedo et al. 275 \\
\hline \multirow[t]{4}{*}{ Malvaceae } & Abutilon Mill. sp. & $\mathrm{AB}$ & NMA & M.A. Milward-de-Azevedo et al. 265 \\
\hline & Eriotheca Schott \& Endl. sp. & AR & NMA & M.A. Milward-de-Azevedo et al. 223 \\
\hline & Guazuma Adans sp. & AR & NMA & M.A. Milward-de-Azevedo et al. 318 \\
\hline & Helicteres sacarolha St.-Hil., Juss. \& Camb. & SB & NMA & $\begin{array}{l}\text { M.A. Milward-de-Azevedo et al. } \\
249,441\end{array}$ \\
\hline
\end{tabular}




\begin{tabular}{|c|c|c|c|c|}
\hline Família & Gênero / Espécie & Hábito & Origem & Voucher \\
\hline & Sida acuta Burm. & $\mathrm{HE}$ & NMA & $\begin{array}{l}\text { M.A. Milward-de-Azevedo et al. } \\
\text { 382, } 394\end{array}$ \\
\hline & Sida cordifolia $\mathrm{L}$. & $\mathrm{AB}$ & NMA & M.A. Milward-de-Azevedo et al. 326 \\
\hline & Triumfetta semitriloba Jacq. & SB & NMA & $\begin{array}{l}\text { M.A. Milward-de-Azevedo et al. } \\
\text { 245, 248, 266, 310, } 328\end{array}$ \\
\hline \multirow[t]{8}{*}{ Melastomataceae } & Clidemia hirta (L.) D. Don & $\mathrm{AB}$ & NMA & $\begin{array}{l}\text { M.A. Milward-de-Azevedo et al. } \\
\text { 383, 393, } 425\end{array}$ \\
\hline & * Clidemia allardii Wurdack & $\mathrm{AB}$ & EX-MA & M.A. Milward-de-Azevedo et al. 222 \\
\hline & \# Leandra nianga Cogn. & $\mathrm{AB}$ & NMA & $\begin{array}{l}\text { M.A. Milward-de-Azevedo et al. } \\
341,345\end{array}$ \\
\hline & Leandra reversa (DC.) Cogn. & $\mathrm{AB}$ & NMA & M.A. Milward-de-Azevedo et al. 239 \\
\hline & Miconia albicans (Sw.) Steud. & SB & NMA & M.A. Milward-de-Azevedo et al. 352 \\
\hline & \# Miconia ibaguensis (Bonpl.) Triana & SB & NMA & $\begin{array}{l}\text { M.A. Milward-de-Azevedo et al. } \\
225,302,347,350,420\end{array}$ \\
\hline & Ossaea DC. sp. & SB & NMA & M.A. Milward-de-Azevedo et al. s.n. \\
\hline & Tibouchina Abl. & SB & NMA & M.A. Milward-de-Azevedo et al. 340 \\
\hline \multirow[t]{4}{*}{ Meliaceae } & $\Delta$ Cedrela fissilis Vell. & AR & NMA & M.A. Milward-de-Azevedo et al. s.n. \\
\hline & Guarea guidonia (L.) Sleumer & $\mathrm{AR}$ & NMA & $\begin{array}{l}\text { M.A. Milward-de-Azevedo et al. } \\
315,387,444\end{array}$ \\
\hline & Trichilia P. Broune sp. & $\mathrm{AR}$ & NMA & M.A. Milward-de-Azevedo et al. 454 \\
\hline & Trichilia pallida Sw. & $\mathrm{AR}$ & NMA & M.A. Milward-de-Azevedo et al. 253 \\
\hline \multirow[t]{3}{*}{ Moraceae } & Brosimum Sw. sp. & $\mathrm{AR}$ & NMA & M.A. Milward-de-Azevedo et al. 354 \\
\hline & Ficus elastica Roxb. ex Hornem. & $\mathrm{AR}$ & EX-BR & M.A. Milward-de-Azevedo et al. s.n. \\
\hline & Ficus gomelleira Kunth \& C.D. Bouché & $\mathrm{AR}$ & NMA & M.A. Milward-de-Azevedo et al. s.n. \\
\hline Musaceae & Musa paradisiaca $\mathrm{L}$. & $\mathrm{AB}$ & EX-BR & M.A. Milward-de-Azevedo et al. s.n. \\
\hline \multirow[t]{8}{*}{ Myrtaceae } & * Eugenia jambolana Lam. & AR & EX-BR & M.A. Milward-de-Azevedo et al. s.n. \\
\hline & Eugenia uniflora $\mathrm{L}$. & $\mathrm{AB}$ & NMA & M.A. Milward-de-Azevedo et al. 334 \\
\hline & Eugenia L. sp. & $\mathrm{AR}$ & NMA & M.A. Milward-de-Azevedo et al. 424 \\
\hline & Jambosa DC. sp. & $\mathrm{AR}$ & NAT & M.A. Milward-de-Azevedo et al. s.n. \\
\hline & \# * Myrcia floridissima Sobral & $\mathrm{AR}$ & NMA & $\begin{array}{l}\text { M.A. Milward-de-Azevedo et al. } \\
\text { 285, 311, } 369\end{array}$ \\
\hline & Myrcia tomentosa (Aubl.) DC. & $\mathrm{AR}$ & NMA & M.A. Milward-de-Azevedo et al. s.n. \\
\hline & Psidium guajava $\mathrm{L}$. & $\mathrm{AB}$ & NMA & M.A. Milward-de-Azevedo et al. s.n. \\
\hline & Syzygium cumini (L.) Skeels & $\mathrm{AR}$ & NAT & M.A. Milward-de-Azevedo et al. s.n. \\
\hline Ochnaceae & $\begin{array}{l}\text { \# * Ouratea spectabilis (Mart. ex Engl.) } \\
\text { Engl. }\end{array}$ & $\mathrm{AB}$ & EX-MA & M.A. Milward-de-Azevedo et al. s.n. \\
\hline Oxalidaceae & Oxalis L. sp. & $\mathrm{HE}$ & NMA & M.A. Milward-de-Azevedo et al. 426 \\
\hline Passifloraceae & \# Passiflora alata Curtis & $\mathrm{TP}$ & NMA & $\begin{array}{l}\text { M.A. Milward-de-Azevedo et al. } \\
286,434\end{array}$ \\
\hline
\end{tabular}




\begin{tabular}{|c|c|c|c|c|}
\hline Família & Gênero / Espécie & Hábito & Origem & Voucher \\
\hline & Passiflora amethystina Mikan & $\mathrm{TP}$ & NMA & $\begin{array}{l}\text { M.A. Milward-de-Azevedo et al. } \\
\text { 261, 439, K.F. Borges } 01 \text { et H.G. } \\
\text { Castro }\end{array}$ \\
\hline & Passiflora capsularis L. & $\mathrm{TP}$ & NMA & $\begin{array}{l}\text { M.A. Milward-de-Azevedo et al. } \\
\text { 262, 313, 319, 323, 401, K.F. } \\
\text { Borges } 04 \text { et H.G. Castro }\end{array}$ \\
\hline & Passiflora edulis Sims & $\mathrm{TP}$ & NMA & M.A. Milward-de-Azevedo et al. 403 \\
\hline & Passiflora porophylla Vell. & $\mathrm{TP}$ & NMA & $\begin{array}{l}\text { M.A. Milward-de-Azevedo et al. } \\
\text { 467, K.F. Borges et H.G. Castro } 07\end{array}$ \\
\hline & \# Passiflora speciosa Gardn. & $\mathrm{TP}$ & NMA & $\begin{array}{l}\text { M.A. Milward-de-Azevedo et al. } \\
\text { 299, K.F. Borges } 09\end{array}$ \\
\hline \multirow[t]{10}{*}{ Piperaceae } & Piper arboreum Aubl. & $\mathrm{AR}$ & NMA & M.A. Milward-de-Azevedo et al. 365 \\
\hline & Piper aduncum L. & $\mathrm{AB}$ & NMA & M.A. Milward-de-Azevedo et al. 233 \\
\hline & Piper amalago L. & $\mathrm{AB}$ & NMA & M.A. Milward-de-Azevedo et al. 430 \\
\hline & \# Piper corcovadensis (Miq.) C. DC. & $\mathrm{HE}$ & NMA & M.A. Milward-de-Azevedo et al. 371 \\
\hline & * Piper frutescens C. DC. & $\mathrm{AB}$ & NMA & M.A. Milward-de-Azevedo et al. 343 \\
\hline & Piper hispidum Sw. & $\mathrm{AB}$ & NMA & M.A. Milward-de-Azevedo et al. 320 \\
\hline & Piper mollicomum Kunth & $\mathrm{AB}$ & NMA & $\begin{array}{l}\text { M.A. Milward-de-Azevedo et al. } \\
\text { 339, } 374\end{array}$ \\
\hline & \# Piper rivinoides Kunth & $\mathrm{AB}$ & NMA & M.A. Milward-de-Azevedo et al. 431 \\
\hline & Piper umbellatum (L.) Miq. & SB & NMA & $\begin{array}{l}\text { M.A. Milward-de-Azevedo et al. } \\
\text { 240, } 405\end{array}$ \\
\hline & \# Piper xylosteoides (Kunth.) Steud. & SB & NMA & M.A. Milward-de-Azevedo et al. 379 \\
\hline \multirow[t]{2}{*}{ Plantaginaceae } & * Plantago lanceolata L. & $\mathrm{HE}$ & NAT & M.A. Milward-de-Azevedo et al. s.n. \\
\hline & Plantago major L. & $\mathrm{HE}$ & NAT & M.A. Milward-de-Azevedo et al. s.n. \\
\hline \multirow[t]{9}{*}{ Poaceae } & Andropogon bicornis L. & $\mathrm{HE}$ & NMA & M.A. Milward-de-Azevedo et al. s.n. \\
\hline & * Bambusa vulgaris Schrad. ex J.C. Wendl. & $\mathrm{AB}$ & NAT & M.A. Milward-de-Azevedo et al. 231 \\
\hline & Brachiaria (Trin.) Griseb. sp. & $\mathrm{HE}$ & NAT & M.A. Milward-de-Azevedo et al. s.n. \\
\hline & Eleusine indica (L.) Gaertn. & $\mathrm{HE}$ & NAT & M.A. Milward-de-Azevedo et al. s.n. \\
\hline & Hyparrhenia rufa (Nees) Stapf & $\mathrm{HE}$ & NAT & M.A. Milward-de-Azevedo et al. s.n. \\
\hline & $\begin{array}{l}\text { Lasiacis sorghoidea (Desv. ex Ham.) } \\
\text { Hitchc. \& Chase }\end{array}$ & $\mathrm{HE}$ & NMA & M.A. Milward-de-Azevedo et al. 231 \\
\hline & Melinis minutiflora P. Beauv. & $\mathrm{HE}$ & NAT & M.A. Milward-de-Azevedo et al. s.n. \\
\hline & * Panicum maximum Jacq. & $\mathrm{HE}$ & NAT & M.A. Milward-de-Azevedo et al. s.n. \\
\hline & Saccharum L. sp. & $\mathrm{AB}$ & NMA & M.A. Milward-de-Azevedo et al. s.n. \\
\hline Polygalaceae & \# Polygala cyparissias A. St.-Hil. \& Moq. & $\mathrm{HE}$ & NMA & M.A. Milward-de-Azevedo et al. s.n. \\
\hline Primulaceae & Myrsine guianensis (Aubl.) Kuntze & AR & NMA & $\begin{array}{l}\text { M.A. Milward-de-Azevedo et al. } \\
\text { 400; D.R. Couto et al. } 1749 \text { (VIES) }\end{array}$ \\
\hline Rhamnaceae & \# Reissekia smilacina (Sm.) Steud. & $\mathrm{AB}$ & NMA & M.A. Milward-de-Azevedo et al. s.n. \\
\hline Rosaceae & Eriobotrya japonica (Thunb.) Lindl. & $\mathrm{AB}$ & NAT & M.A. Milward-de-Azevedo et al. s.n. \\
\hline
\end{tabular}




\begin{tabular}{|c|c|c|c|c|}
\hline Família & Gênero / Espécie & Hábito & Origem & Voucher \\
\hline & Rubus rosifolius $\mathrm{Sm}$. & $\mathrm{HE}$ & NMA & $\begin{array}{l}\text { M.A. Milward-de-Azevedo et al. } \\
245,317,397\end{array}$ \\
\hline \multirow[t]{10}{*}{ Rubiaceae } & Borreria G. Mey. sp. & $\mathrm{HE}$ & NMA & M.A. Milward-de-Azevedo et al. s.n. \\
\hline & Borreria verticillata (L.) G.Mey. & SB & NMA & M.A. Milward-de-Azevedo et al. 305 \\
\hline & $\begin{array}{l}\text { * Coccocypselum aureum (Spreng.) } \\
\text { Cham. \& Schltdl. }\end{array}$ & $\mathrm{HE}$ & NMA & M.A. Milward-de-Azevedo et al. 357 \\
\hline & Coffea L. sp. & $\mathrm{AB}$ & NAT & M.A. Milward-de-Azevedo et al. s.n. \\
\hline & Genipa americana $\mathrm{L}$. & AR & NMA & M.A. Milward-de-Azevedo et al. s.n. \\
\hline & Geophila repens (L.) I.M. Johnst. & $\mathrm{HE}$ & NMA & M.A. Milward-de-Azevedo et al. 256 \\
\hline & \#* Manettia pubescens Cham. \& Schltdl. & $\mathrm{TP}$ & NMA & M.A. Milward-de-Azevedo et al. 244 \\
\hline & Psychotria L. & SB & NMA & M.A. Milward-de-Azevedo et al. 377 \\
\hline & s Rudgea jasminoides (Cham.) Müll. Arg. & $\mathrm{AR}$ & NMA & M.A. Milward-de-Azevedo et al. s.n. \\
\hline & Spermacoce verticillata $\mathrm{L}$. & $\mathrm{HE}$ & NMA & M.A. Milward-de-Azevedo et al. s.n. \\
\hline Rutaceae & * Citrus limonum Risso & AR & EX-BR & M.A. Milward-de-Azevedo et al. s.n. \\
\hline Salicaceae & Prockia crucis P. Browne ex L. & $\mathrm{AR}$ & NMA & $\begin{array}{l}\text { M.A. Milward-de-Azevedo et al. } \\
318,370\end{array}$ \\
\hline \multirow[t]{3}{*}{ Sapindaceae } & Allophylus racemosus Radlk. & $\mathrm{AB}$ & NMA & $\begin{array}{l}\text { M.A. Milward-de-Azevedo et al. } \\
364,380\end{array}$ \\
\hline & Cupania L. sp. & AR & NMA & M.A. Milward-de-Azevedo et al. s.n. \\
\hline & Sapindus saponaria L. & AR & NMA & M.A. Milward-de-Azevedo et al. s.n. \\
\hline Sapotaceae & $\begin{array}{l}\text { * Pouteria sapota (Jacq.) } \\
\text { H.E. Moore \& Stearn }\end{array}$ & $\mathrm{AR}$ & EX-BR & M.A. Milward-de-Azevedo et al. s.n. \\
\hline Siparunaceae & Siparuna guianensis Aubl. & $\mathrm{AB}$ & NMA & $\begin{array}{l}\text { M.A. Milward-de-Azevedo et al. } \\
346,368\end{array}$ \\
\hline Smilacaceae & Smilax L. sp. & LI & NMA & M.A. Milward-de-Azevedo et al. 232 \\
\hline \multirow[t]{3}{*}{ Solanaceae } & Cestrum intermedium Sendtn. & AR & NMA & M.A. Milward-de-Azevedo et al. s.n. \\
\hline & Solanum americanum Mill. & $\mathrm{AB}$ & NMA & M.A. Milward-de-Azevedo et al. s.n. \\
\hline & Solanum viarum Dunal & $\mathrm{HE}$ & NMA & M.A. Milward-de-Azevedo et al. 406 \\
\hline \multirow[t]{5}{*}{ Urticaceae } & Bohemeria caudata $\mathrm{Sw}$. & $\mathrm{AB}$ & NMA & M.A. Milward-de-Azevedo et al. 322 \\
\hline & \# Cecropia hololeuca Miq. & AR & NMA & M.A. Milward-de-Azevedo et al. s.n. \\
\hline & Cecropia pachystachya Trécul & AR & NMA & M.A. Milward-de-Azevedo et al. s.n. \\
\hline & Coussapoa Aubl. & $\mathrm{AR}$ & NMA & M.A. Milward-de-Azevedo et al. 277 \\
\hline & Phenax Wedd. & SB & NMA & M.A. Milward-de-Azevedo et al. 243 \\
\hline \multirow[t]{2}{*}{ Verbenaceae } & Lantana camara $\mathrm{L}$. & $\mathrm{AB}$ & NAT & $\begin{array}{l}\text { M.A. Milward-de-Azevedo et al. } \\
\text { 215, 330, 333, 398, } 436\end{array}$ \\
\hline & Stachytarpheta cayennensis (Rich) Vahl & $\mathrm{HE}$ & NMA & $\begin{array}{l}\text { M.A. Milward-de-Azevedo et al. } \\
\text { 241, } 365\end{array}$ \\
\hline Violaceae & Anchietea pyrifolia (Mart.) G.Don & $\mathrm{TP}$ & NMA & M.A. Milward-de-Azevedo et al. 308 \\
\hline \multirow[t]{2}{*}{ Zingiberaceae } & * Curcuma roscoeana Wall. & $\mathrm{HE}$ & EX-BR & M.A. Milward-de-Azevedo et al. 433 \\
\hline & Hedychium coronarium J. Köing & $\mathrm{HE}$ & NAT & M.A. Milward-de-Azevedo et al. 446 \\
\hline
\end{tabular}


ressaltam o grande número de EEI nas Unidades de Conservação da Mata Atlântica, devido a este ter sido o primeiro bioma a ser colonizado pelos europeus, possuindo as maiores concentrações humanas e as atividades agropastoris mais antigas do Brasil, logo, representa o bioma mais degradado e com menor área nativa conservada, sendo o mais afetado por EEI, dentro e fora de Unidades de Conservação.

A grande influência antrópica nos entornos e dentro da área do PECF interfere na vegetação, o que levou a coleta de diferentes espécies com potencial invasor, sendo a maioria representantes da família Poaceae, sendo elas: Andropogon bicornis L. (capimrabo-de-burro), e as de origem africana Hyparrhenia rufa (Nees) Stapf(capim-jaraguá), Melinis minutiflora P. Beauv. (capim-gordura) e Panicum maximum Jacq. (capim-colonião), utilizados para formação de pastagens (Matos \& Pivello 2009). Nos entornos do PECF são encontradas diversas propriedades com atividade pecuária, o que pode ter elevado a taxa de disseminação dessas espécies para dentro do PECF. Panicum maximum, considerada invasora agressiva, apresenta potencial de deslocar até mesmo as espécies Melinis minutiflora e Hyparrhenia rufa (Pivello 2011), outras duas espécies daninhas encontradas no PECF. Além das espécies gramíneas, algumas outras consideradas naturalizadas podem causar grande impacto na biodiversidade local, como é o caso de Coffea sp. e Tecoma stans (L.) Juss. ex Kunth, as quais competem com espécies nativas nos estratos inferiores de formações florestais, interferindo no processo de regeneração natural e de sucessão desses remanescentes (IABIN 2017).

As espécies invasoras podem tornar-se um grave problema à biodiversidade local, uma vez que possuem elevada capacidade reprodutiva e dispersão, levando a uma maior competição com a população nativa e também formam uma espessa camada de biomassa no solo, propiciando a redução da luminosidade na superfície do solo, podendo assim evitar a germinação de espécies nativas que necessitam de luminosidade para a quebra de dormência de suas sementes (Pivello 2011). E ainda, por extraírem grande quantidade de nutrientes para o seu crescimento, podem propiciar alterações nos ciclos de nutrientes no solo (Asner \& Beatty 1996).

A análise temporal da cobertura vegetal do PECF, realizado por Souza et al. (2015), utilizando a técnica de sensoriamento remoto por intermédio do índice de vegetação da diferença normalizada, entre os anos de 1985 e 2010 , demonstrou que cerca de $71 \%$ de sua área não sofreu alteração, 20\% foi regenerado e $9 \%$ tiveram processos de desmatamentos devido à ação antrópica realizada pelos proprietários rurais vizinhos, como o plantio de café e a criação de gado. Dados similares foram encontrados por Thuler et al. (2012), ao analisar imagens da LANDSAT de 1996 e 2010. Esses resultados sugerem que mesmo com a ação antrópica no PECF e ao entorno do mesmo, o número de espécies nativas de Mata atlântica é bastante elevado. O que vem a ser corroborado com esse estudo, pois foi verificado que cerca $80 \%$ das espécies amostradas são nativas de Mata Atlântica, demonstrando assim que o PECF sofreu pouca alteração vegetacional.

Entre as espécies listadas neste inventário, segundo a Resolução no 29 (CONAMA 1994) que define a vegetação primária e secundária nos estágios inicial, médio e avançado de regeneração da Mata Atlântica, foram encontradas como representantes de vegetação primária as espécies Cariniana Casar. sp., Piptadenia gonoacantha (Mart.) J.F. Macbr., Tabebuia heptaphylla (Vell.) Toledo e Tabebuia serratifolia (Vahl) G. Nicholson. As espécies secundárias foram Anacardium occidentale L., Cecropia hololeuca Miq., Cecropia pachystachya Trécul, Cedrela fissilis Vell., Psidium guajava L. e Vernonanthura polyantes (Sprengel) Vega \& Dematteis. Esse número é pequeno em comparação com o montante de espécies descritas nesse inventário, o que leva a afirmativa de que o PECF apresenta pequenas áreas em estágio de regeneração, como descrito por Souza et al. (2015), onde os autores afirmam que 20,11\% da área sofreram processos de regeneração e 71,18\% da vegetação não sofreram mudanças desde a criação do parque.

Mesmo com a contribuição desse levantamento florístico a fim de complementar os registros de espécies ocorrentes em remanescentes de Floresta Atlântica no Sul do Espírito Santo, diversas áreas não apresentam dados de coletas. Os trabalhos realizados no estado são quase que exclusivos de parques e reservas biológicas (Oliveira et al. 2013; Luber et al. 2016; Souza et al. 2016). Futuros inventários florísticos, em áreas com lacunas de coletas são fundamentais para o enriquecimento sobre a flora do estado, além do acréscimo no banco de dados sobre as espécies vegetais ocorrentes na Mata Atlântica, pois estes estudos apresentam a amplitude de ocorrência das espécies, e essa variação pode ser explicada pelo tipo de clima e pluviosidade da região, tamanho das áreas amostradas, e também das metodologias adotadas para a estimativa da diversidade.

Este trabalho apresentou caráter pioneiro na área de estudo e poderá ser utilizado como base para futuros levantamentos e atualizações quanto à 
flora local, além de apontar uma riqueza de espécies no remanescente pertencente ao PECF. Estes dados mostram-se importantes para a realização de políticas públicas que busquem a conservação das espécies vegetais. $\mathrm{O}$ número de táxons encontrados no $\mathrm{PECF}$ indica o potencial de pequenos fragmentos florestais serem levados em consideração na elaboração de planos de conservação.

\section{Agradecimentos}

Aos pesquisadores e especialistas, a identificação do material; em especial a Gustavo Shimizu, Gustavo Heinden, José Fernando A. Baumgratz, Micheline Carvalho-Silva e Ronaldo Marquete, o precioso auxílio.

\section{Referências}

Asner GP \& Beatty SW (1996) Effects of an African grass invasion on Hawaian shrubland nitrogen biogeochemistry. Plant \& Soil 186: 205-211.

Assis AM, Thomaz LD \& Pereira OJ (2004) Florística de um trecho de floresta de restinga no município de Guarapari, Espírito Santo, Brasil. Acta Botanica Brasilica 18: 191-201.

BFG - The Brazil Flora Group (2015) Growing knowledge: an overview of seed plant diversity in Brazil. Rodriguésia 66: 1085-1113.

Brooks DR, Mayden RL \& McLennan DA (1992) Phylogeny and biodiversity: conserving our evolutionary legacy. Trends in Ecology and Evolution 7: 55-59.

Canhos VP, Souza S, Giovanni RD \& Canhos DAL (2004) Global biodiversity informatics: setting the scene for a "new world" of ecological modeling. Biodiversity Informatics 1: 1-13.

Chagas AP, Peterle PL, Thomaz LD, Dutra VF \& Valadares RT (2014) Leguminosae - Caesalpinioideae do Parque Estadual Paulo César Vinha, Espírito Santo, Brasil. Rodriguésia 65: 99-112.

CNCFlora - Centro Nacional de Conservação da Flora (2017) Disponível em <http://cncflora.jbrj.gov. br/portal/pt-br/profile/Rudgea jasminoides subsp. nervosa $>$. Acesso em 11 março 2017.

CONAMA - Conselho Nacional do Meio Ambiente (1994) Resolução no 29, de 7 de dezembro de 1994. Disponível em <http://www.mma.gov.br/port/ conama/res/res94/res2994.html>. Acesso em 11 maio 2017.

Couto DR, Francisco TM, Manhães VC, Dias HM \& Pereira MCA (2017) Floristic composition of a Neotropical inselberg from Espírito Santo state, Brazil: an important area for conservation. Check List 13: 2043. DOI: https://doi.org/10.15560/13.1.2043

Dutra VF, Alves-Araújo A\& Carrijo TT (2015)Angiosperm checklist of Espírito Santo: using electronic tools to improve the knowledge of an Atlantic Forest biodiversity hotspot. Rodriguésia 66: 1145-1152. DOI: $10.1590 / 2175-7860201566414$

Fabris LC \& Peixoto AL (2013) Sapotaceae das restingas do Espírito Santo, Brasil. Rodriguésia 64: 263-283.

Font Quer P (1992) Diccionario de botánica. 7 ${ }^{\mathrm{a}}$ ed. Labor, Barcelona. 1280p.

Fraga CN, Simonelli M \&. Fernandes HQB (2007) Metodologia utilizada na elaboração da lista da flora ameaçada de extinção no Espírito Santo. In: Fraga CN \& Simonelli M (orgs.) Espécies da flora ameaçadas de extinção no estado Espírito Santo. IPEMA, Vitória. Pp. 59-72.

Galindo-Leal C \& Câmara IG (2005) Status do hotspot Mata Atlântica: uma síntese. In: Galindo-Leal C \& Câmara IG (eds.) Mata Atlântica: biodiversidade, ameaças e perspectivas. Fundação SOS Mata Atlântica, São Paulo. Pp. 3-9.

Hernández L, Dezzeo N, Sanoja E, Salazar L \& Castellanos $H$ (2012) Changes in structure and composition of evergreen forests on an altitudinal gradient in the Venezuelan Guayana shield. Revista de Biología Tropical 60: 11-33.

IABIN - Rede Inter Americana de Informação sobre Biodiversidade (2017) Base de dados nacional de espécies exóticas invasoras I3N Brasil. Instituto Hórus de Desenvolvimento e Conservação Ambiental. Florianópolis, SC. Disponível em $<$ http://i3n. institutohorus.org.br/www $>$. Acesso em 10 maio 2017.

IDAF - Instituto de Defesa Agropecuária e Florestal do Espírito Santo (2000) Plano de manejo do Parque Estadual Cachoeira da Fumaça. MMA, FNMA, Vitória. 42p.

IEMA - Instituto Estadual de Meio Ambiente e Recursos Hídricos (2017) Parque Estadual Cachoeira da Fumaça. Disponível em $<$ https://iema.es.gov.br/ Media/iema/Downloads/GRN/2017.10.05\%20-\%20 Plano\%20Emergencial\%20UP PECF.pdf $>$. Acesso em 12 fevereiro 2017.

Lima JR, Sampaio EVSB, Rodal MJN \& Araújo FS (2009) Composição florística da floresta estacional decídua montana de Serra das Almas, CE, Brasil. Acta Botanica Brasilica 23: 756-763.

Luber J, Tuler AC, Torres F, Christ JA, Guidoni-Martins KG, Zanetti M, Hollunder RK, Manhães VC, Zorzanelli JPF, Mendonça ES, Garbin ML \& Carrijo TT (2016) List of angiosperm species in an Atlantic Forest fragmente reveals collection gaps in Espírito Santo state, Brazil. Check List 12: 1835.

Martinelli G \& Moraes MA(2013) Livro vermelho da flora do Brasil. Andrea Jacobsson, Instituto de Pesquisas Jardim Botânico do Rio de Janeiro, Rio de Janeiro. 1100 p.

Martini AMZ, Fiaschi P, Amorim AM \& Paixão JL (2007) A hotpoint within a hot-spot: a high diversity site in Brazil's Atlantic forest. Biodiversity and Conservation 16: 3111-3128. 
Matos DMS \& Pivello VR (2009) O impacto das plantas invasoras nos recursos naturais de ambientes terrestres - alguns casos brasileiros. Ciência e Cultura 61: 27-30.

Metzger J (2003) Como restaurar a conectividade de paisagens fragmentadas? In: Kageyama P, Oliveira R, Moraes L, Engel V \& Gandara F (orgs.) Restauração ecológica de ecossistemas naturais. Fundação de Estudos e Pesquisas Agrícolas Florestais, Botucatu. Pp. 49-76.

Metzger JP (2009) Conservation issues in the Brazilian Atlantic forest. Biological Conservation 142: 11381140 .

MMA - Ministério do Meio Ambiente (2014) Portaria do Ministério do Meio Ambiente $\mathrm{n}^{\circ} 443$, de 17 de dezembro de 2014. Disponível em <http:// cncflora.jbrj.gov.br/portal/static/pdf/portaria mma_443_2014.pdf >. Acesso em 1 março 2017.

Myers N, Mittermeier RA, Mittermeier CG, Fonseca GAB \& Kent J (2000) Biodiversity hotspots for conservation priorities. Nature 403: 853-858.

Oliveira BR, Bravo VJ, Bravo MA \& Franco BKS (2013) Florística e fitossociologia de uma Floresta Ombrófila Densa, Santa Teresa, Espírito Santo, Brasil. Natureza on line 11: 187-192.

Pereira OJ (2007) A cobertura vegetal do Espírito Santo. In: Simonelli M \& Fraga CN (orgs.) Espécies da flora ameaçadas de extinção no estado do Espírito Santo. IPEMAP, Vitória. Pp. 17-20.

Pereira OJ, Assis AM \& Souza RLD (1998) Vegetação da restinga de Pontal do Ipiranga, Município de Linhares (ES). Anais do IV Simpósio de Ecossistemas Brasileiros 3: 117-128.

Pereira OJ \& Araujo DSD (2000) Análise florística das restingas dos estados do Espírito Santo e Rio de Janeiro. In: Esteves FA \& Lacerda LD (eds.) Ecologia de restingas e lagoas costeiras. NUPEM/UFRJ, Macaé. Pp. 25-63.

Peterle PL, Chagas AP, Thomaz LD, Dutra VF \& Valadares RT (2015) Mimosoideae (Leguminosae) do Parque Estadual Paulo César Vinha, Espírito Santo, Brasil. Rodriguésia 66: 245-257.

Pillar VD, Müller SC, Castilhos Z \& Jacques AVA (2009) Campos Sulinos: conservação e uso sustentável da biodiversidade. Ministério do Meio Ambiente, Brasília. 403p.

Pitman NCA, Terborgh JW, Silman MR, Núñez VP, Neill DA, Cerón CE, Palacios WA \& Aulestia M (2002) A comparison of tree species diversity in two upper Amazonian forests. Ecology 83: 3210-3224.

Pivello VR (2011) Invasões biológicas no cerrado brasileiro. Efeito da introdução de espécies exóticas sobre a biodiversidade. Ecologia Info 33. Disponível em $<$ http://ecologia.info/cerrado.htm $>$. Acesso em 10 maio 2017.

Ribeiro MC, Metzger JP, Martensen AC, Ponzoni F \& Hirota MM (2009) Brazilian Atlantic forest: how much is left and how is the remaining forest distributed? Implications for conservation. Biological Conservation 142: 1141-1153.

Saiter FZ, Wendt T, Villela DM \& Nascimento MT (2008) Rain Forest: Floristics. In: Del Claro K, Oliveira PS \& Rico-Gray V (org.) Tropical biology and conservation management. Unesco, Paris. Pp. 203-228

Saiter FZ \& Thomaz LD (2014) Revisão da lista de espécies arbóreas do inventário de Thomaz \& Monteiro (1997) na Estação Biológica de Santa Lúcia: o mais importante estudo fitossociológico em florestas montanas do Espírito Santo. Boletim do Museu de Biologia Mello Leitão 34: 101-128.

Sampaio AB \& Schmidt IB (2013) Espécies exóticas invasoras em unidades de conservação federais do Brasil. Biodiversidade Brasileira 3: 32-49.

Sarnaglia Junior VB, Thomaz LD \& Guimarães EF (2014) O gênero Peperomia Ruiz \& Pav. na Área de Proteção Ambiental do Mestre Álvaro, Espírito Santo, Brasil. Boletim do Museu de Biologia Mello Leitão 35: 21-34.

Souza MC, Kawakita K, Slusarski SR \& Pereira, GF (2009) Vascular flora of the Upper Paraná River floodplain. Brazilian Journal of Biology 69: 735-745.

Souza GSA, Conrado VN, Martins BF, Quinto VM \& Silva E (2015) Análise temporal da cobertura vegetal no Parque Estadual Cachoeira da Fumaça, ES, por meio de técnicas de sensoriamento remoto. Revista Verde de Agroecologia e Desenvolvimento Sustentável 10: 103-107.

Souza WO, Machado JO, Tognella MMP \& Araújo AA (2016) Checklist de angiospermas do Parque Estadual de Itaúnas, Espírito Santo, Brasil. Rodriguésia 67: 571-581.

Tabarelli M, Pinto LP, Silva JMC, Hirota M \& Bedê L (2005) Challenges and opportunities for biodiversity conservation in the Brazilian Atlantic Forest. Conservation Biology 19: 695-700.

The Plant List (2017) Disponível em <http://www. theplantlist.org/>. Acesso em 11 março 2017.

Thiers B [continuously updated]. Index Herbariorum: a global directory of public herbaria and associated staff. New York Botanical Garden's Virtual Herbarium. Disponível em $<$ http://sweetgum.nybg.org/science/ $\mathrm{ih} />$. Acesso em 12 fevereiro 2017.

Thuler LHM, Quinto VM, Bernardes PM, Beltrame RA \& Fonseca AS (2012) Estudo da dinâmica florestal no Parque Estadual Cachoeira da Fumaça por meio de subtração de imagens NDVI. Enciclopédia Biosfera 8: 1251-1262. 